\title{
A New Method for Olive Fruits Recognition
}

\author{
C. Gabriel Gatica ${ }^{1,2}$, S. Stanley Best ${ }^{2}$, José Ceroni $^{3}$, and Gaston Lefranc ${ }^{1}$ \\ 1 Pontificia Universidad Católica de Valparaíso, Chile, \\ Escuela de Ingeniería Eléctrica \\ gabriel.gatica@hotmail.com, glefranc@gmail.com \\ 2 Instituto de Investigaciones Agropecuarias, \\ INIA, PROGAP, Chillán, Chile \\ sbest@inia.cl \\ 3 Pontificia Universidad Católica de Valparaíso, Chile, \\ Escuela de Ingeniería Industrial \\ jceroni@ucv.cl
}

\begin{abstract}
A model for the recognition of the diameter of olives is presented. The information regarding size of olive fruits is intended for estimating the best harvesting time of olive trees. The recognition is performed by analyzing the RGB images obtained from olive tree pictures
\end{abstract}

Keywords: image processing, pattern recognition, RGB model, CIELAB color space, olive harvesting.

\section{Introduction}

One of the important aspects in olive cultivation is being able to estimate when the harvest should take place, trying to obtain the best yielding from the land. Based on this information, it is possible to make the right decisions both financially and agriculturally. The problem of estimating the optimal plantation yield consists on determining the largest fruit size based on its equatorial and polar diameters.

Literature review for the past 30 years shows that there are no publications relating to the count of olives (olive fruit) but only to the identification of olive trees (trees). Most of the publications found in the identification field refer to particular objects. Therefore, we propose to use simultaneously identification and caliber tools to identify the best harvesting time.

Saito et al. 11 used a method of classification of eggplants by using neural networks in the RGB color model and color space CIELAB. The method described by Martinez-Uso et al. 11, uses an algorithm for the minimization of energy for active contours, in order to distinguish the different areas of the fruit, which is the main criterion for image segmentation. This algorithm tends to achieve a similar result to that obtained by clustering.

Unay and Gosselin [2] compare different types of classifiers for the recognition of defective blocks. SVM algorithm achieved the best results, increasing by around $90 \%$ of correct classification. Cornelius et al. [4] showed that object 
recognition can be achieved by correlating the shape and contours of each shape. In many cases, achieved satisfactory results.

Zhao et al. 6] use color and texture properties for the recognition of red and green apples. This analysis proved successful by including the properties of the co-occurrence matrix jointly with the levels of red, performing segmentation using an analysis of "clustering".

Wijethunga et al. 8], proposed a technique for counting kiwis, based on the segmentation of images using the CIELAB color model and the "Watershed" transformation to count the number of fruits in each image. A more simple method was developed by Li et al. [10], proposed the identification of tomatoes using grayscale image, obtaining a $75 \%$ of correct classification.

Another technique that refers to the invariability of objects with respect to size and rotation is SIFT (Scale Invariant Feature Transform) used by Wan-Gan et al. 9]. They proposed an algorithm based on this transformation in order to solve the problem of fruit identification. The results were developed into a pilot, as it performed well; using test scenarios.

According to Mirisaee and Woo [17, by taking a greater amount of information with both, physical and color parameters of the RGB color model, a k-NN (k-Nearest Neighbors) based on Euclidean distance, classifier can be obtained for fruit classification. Classification results reached $90 \%$ of correct classification. In classification of mature fruits and greens, Jamil et al. [7] used a neuro-fuzzy based classification by taking intensity of the red, green, and blue as a parameter they reached an accuracy level of $73.3 \%$ in the classification of ripe and green.

Alternatively, similarly to the study by Unay and Gosselin [2, Wang et al. 5] present a comparison between artificial neural networks (ANN) and Support Vector Machines (SVP) for apple classification. SVP presented a better result in classification apples, yielding almost $90 \%$ of correct classification for the apple harvest. In detection and classification of fruits, Mohamad et al. 3. performed a study based on the classification of ripe and green based on the histogram feature of the red, green, and blue, and the gray scale. Previous studies about recognition and classification of fruits has been done by team of this paper [12, [16].

The biggest difference between the objectives of the studies cited and the work presented here, is that most of the reviewed research is concerned primarily with the identification of specific fruits, leaving a large margin of slack when developing classification models. In this paper, we propose a model to characterize olive fruits in the tree and measuring the caliber of each specimen.

\section{Background Extraction}

From an olive tree picture, histogram analysis is used to verify the possibility of separation of strata and check the data set under analysis for normality. The strata chosen for analysis are: 1) Olive, 2) Leaf type 1 (dark green), 3) Leaf type 2 (light green), and 4) Stem. Fig. 1.a shows the layers corresponding to each stratum with the background turned white for improved perception. 
Stratification of the data is improved due to the color reference standard; otherwise, the visual differences between the strata would have been slightly noticeable in the case of leaves and olives. Fig. 1.b shows data sets used in the statistical analysis described next. Top left is olive fruit, top right is leaf type 1 , bottom left is leaf type 2 , and bottom right is stem. By visual inspection, it could be inferred that in some images there is some overlap of color in the layers, i.e., some olives (or a part of them) have very similar color.

Analyzing the spectral behavior of all 4 layers for the channels red, green, and blue color of RGB model, hue, saturation, value color of model HSV, $L$, $a, b, a / b$ of CIELAB color space, respectively, it is concluded that the layer segmentation opportunity for olives is more feasible in the matrix $a *$. Fig. 1.c shows the spectrum analysis of channel data to the color space for the four strata (olives, leaves types 1 and 2, and stem). The ideal normal distributions for each stratum were plotted along the empirical curve (thicker line) while keeping the same color for each stratum. Then, a statistical analysis verifies the normality of the data in each stratum because of the data or for each stratum are random variables in the first instance under a normal distribution of data. The probability density function of a normal distributed variable $x_{k}$ is defined as:

$$
\varphi_{\mu_{i}, \sigma_{i}{ }^{2}}(x)=\frac{1}{\sigma_{i} \sqrt{2 \pi}} e^{-\frac{1}{2}\left(\frac{x-\mu_{i}}{\sigma_{i}}\right)^{2}},
$$

where:

$\varphi_{\mu_{i}, \sigma_{i}{ }^{2}}(x)$ : probability density function of the $i$-th stratum

$\mu_{i}$ : arithmetic mean of the $i$-th stratum

$\sigma_{i}:$ standard deviation of the $i$-th stratum

$x_{i}$ : discrete random variable

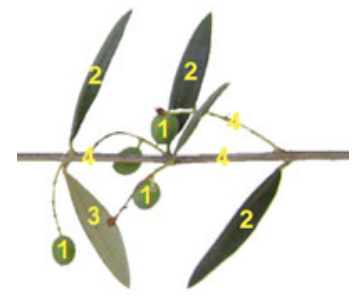

a)

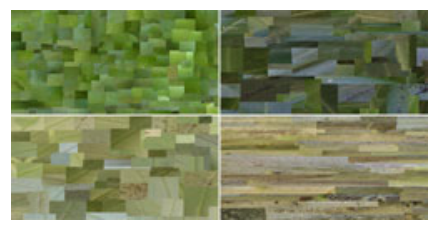

b)

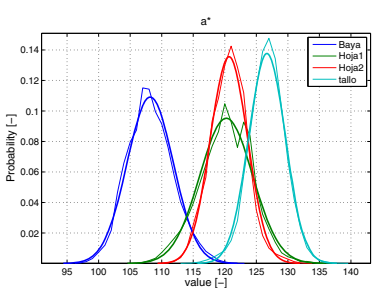

c)

Fig. 1. a) Stratification of the data; b) Set of data used for analysis; c) Spectrum analysis of data

To know the relationship of the data with respect to a normal distribution curve, the random variable is plotted against the probability density curve (normal strata). In this case, the curves obtained have a squared correlation coefficient $R^{2}$ between 0.972 and 0.994 , therefore they could be considered as excellent correlations. To calculate the amount of accumulated data to each point cut in 
the normal distribution curves, it is necessary to integrate the probability density function to that point. The distribution function of a normal distribution is:

$$
\Phi_{\mu_{i}, \sigma_{i}{ }^{2}}\left(x_{c}\right)=\int_{-\infty}^{x_{c}} \Phi_{\mu_{i}, \sigma_{i}{ }^{2}}(t) d t=\int_{-\infty}^{x_{c}} \frac{1}{\sigma_{i} \sqrt{2 \pi}} e^{-\frac{1}{2}\left(\frac{x-\mu_{i}}{\sigma_{i}}\right)^{2}} d t
$$

The main idea of this evaluation is to minimize the amount of probability of occurrence data for the layers leaf type 1 and type two stem leaves, and turn to get as much information as possible for the olives. It is possible to find a good theoretical cutting height, but the data are discrete integer random variables. To resolve this limitation, all distribution functions are evaluated to a closed interval between 112 and 116 to verify their probability of occurrence, Table 1 shows the results.

Table 1. Distribution functions of four strata for closed interval between 112 to 116

\begin{tabular}{|r|c|r|r|r|r|}
\hline \multicolumn{2}{|c|}{ Cutoff value } & \multicolumn{4}{|c|}{ Distribution Function } \\
\hline$x_{c}$ & $x_{c}=f\left(\mu_{1}, \sigma 1\right)$ & Olive & \multicolumn{1}{|c|}{ Leaf 1 } & Leaf 2 & Stem \\
\hline 112 & $\mu_{1}+1.0441 \sigma_{1}$ & 85.1771 & 2.4738 & 0.1620 & 0 \\
113 & $\mu_{1}+1.3176 \sigma_{1}$ & 90.6169 & 4.2190 & 0.4604 & 0 \\
114 & $\mu_{1}+1.5911 \sigma_{1}$ & 94.4198 & 6.8484 & 1.1771 & 0.0008 \\
115 & $\mu_{1}+1.8645 \sigma_{1}$ & 96.8873 & 10.5917 & 2.7126 & 0.0030 \\
116 & $\mu_{1}+2.1380 \sigma_{1}$ & 98.3739 & 15.6276 & 5.6462 & 0.0114 \\
\hline
\end{tabular}

Based on results in Table 1, cutoff value is made equal to 112 . This is because layers of leaf type 1, leaf type 2, and stem together do not exceed $3 \%$ incidence, while stratum corresponding to olives have an incidence of over $85 \%$, having an average cut equal to the mean plus 1.0441 standard deviations.

In order to represent the ideal olive shape, the Principal Components (PCA) method is used. This means to find the best projection that represents our goal (ready to harvest olives) by statistical representation of least squares. The core of this method is to find the eigenvectors or fundamental components of the data sets that shape all possible combinations of olives.

\section{The Model}

Our proposed solution consists in creating three matrices $R_{\text {eigen }}, G_{\text {eigen }}$, and $B_{\text {eigen }}$ respectively, which contain the underlying factors described or as close to it as possible, the data of the stratum or random variables for olives. Having obtained the fundamental matrices linearly independent, theoretically, it is possible to build any olive on the basis of the linear combination of underlying factors represented in the fundamental matrices previously described.

A way to obtain these vectors is by using of PCA method, this method basically find a set of uncorrelated variables to describe the set of observations. In our case, we took a combination of those uncorrelated variables to generate one 


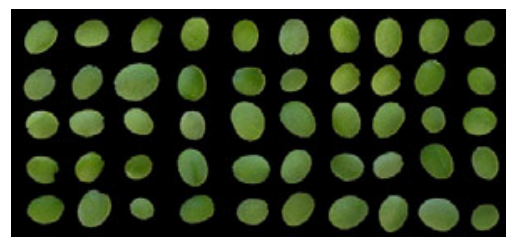

a)

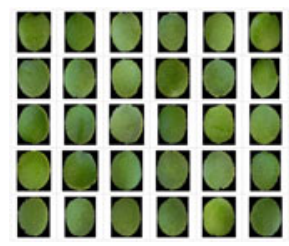

b)

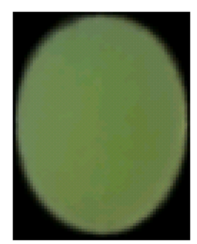

c)

Fig. 2. a) Selected data for analysis; b) Sample of 30 specimens; c) Eigen-image

eigen matrix for each RGB channel. To apply this method, it is necessary to have a data set collected as indicated next. In order to achieve representativeness and also to include variability, samples of various sizes, colors, brightness, contrast, and a number of 50 replications of the random data sets.

Fig. 2.a shows the data set chosen for analysis of principal components. Because olives are of different sizes and their pictures captured at different angles of inclination, a routine to find its rotation angle with respect to a reference point considering the polar and equatorial diameters of the olives was devised. Once found these angles, the median longitudinal, and equatorial diameters, data normalization was applied. Note that the computation of median (or 50 percentile of the data) is independent of presence of extreme data (Fig. 2.b).

After analyzing the 50 standardized samples, for simplicity the eigenvectors were restricted to one dimension for channel, and then this restriction proceeded to merge these three matrices themselves in one of three layers red, green, and blue. Fig. 2.c shows the eigen-image obtained with the PCA analysis.

The cross-correlation index can be used for identifying patterns or correlation between two datasets. An advantage of this index is its utilization with discrete values as well as with continuous functions. It is known that within the CIELAB color space segments of the olives, leaves, and stem are markedly distinct and well defined, as well as with the eigen-image obtained after the PCA process. These two sets of data can also be correlated. The cross-correlation can quickly find sites that correlate better. Figure 3 shows the cross-correlation results.

Regarding olive counting, the need to estimate olives size makes the process not yet complete (contour estimation). Estimation can be achieved by using centroids of figures with greater correlation to the olive's shape, however the

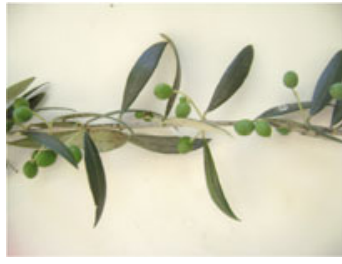

a)

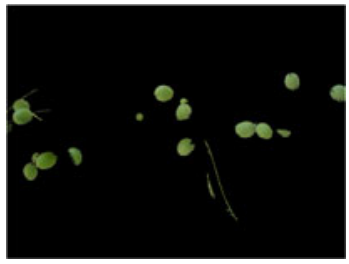

b)

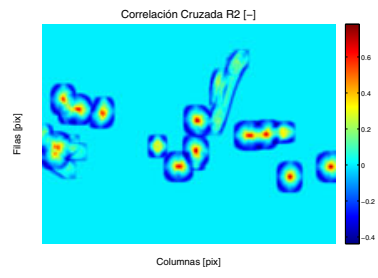

c)

Fig. 3. a) Cross-correlation test; b) filter LAB color space; c) Cross correlation 
olive shapes are variable and not complete. Problems arise when there are: two or more overlapped olives. The solution proposed to this problem is an iterative expansion of the object, starting from the centroid to a boundary that minimizes the energy function. Then, splitting separates contiguous specimens by Euclidean distance transformations, for eliminating possible random seams.

By minimizing the Euclidean distance between the candidate's contours, it is possible to reformulate the problem due to boundary estimation assuming presence of active rather than passive or constant contours (Active Contours Chan-Vese method). This last consideration makes possible to vary the image and also allows us to calculate the gradient. The importance of the gradient is critical because it is a normal vector to the surface under study. The gradient gives a significant value where changes or variations occur, which in consequence is very helpful to finding the contours.

On the other hand, the centroids of the potential candidates were identified by the cross-correlation function. However, the problem is the extent to which adjacent olives overlap among them. According to Stokes theorem [15], it can be assumed that the closed integral, limiting the area of the vector field generated by the gradient, equals the surface integral of the curl of the vector field on an open surface. The value of this integral corresponds to the dot product of the vector field with respect to a differential arc length.

The solution proposed by Chan and Vese [13, is to minimize the energy function required to form a region. This solution was described for solving the Mumford-Shah paradigm [14] 15], resulting in an active solution that iteratively finds the point when the energy function phi is zero. It is the same for both regions, as described also by the Continuity theorem. The energy formula is defined by equation (3), where the terms corresponds to the calculated surface energy a and b, respectively.

$$
\phi=\int_{\Omega_{a}}\left(\hat{I}-\overline{X_{a}}\right)^{2} d A+\int_{\Omega_{b}}\left(\hat{I}-\overline{X_{b}}\right)^{2} d A
$$

Figure Fig. 4.a show the mask generated using the contour search algorithm. One aspect to be emphasized is the starting point of the process of energy minimization. The initial mask of active contours is nothing more than the centroids obtained by the correlation (Fig. 4.b). Note that if iterations tend to infinity or a very large number, it only would slow the overall process, and it also may be incurred in returning to the image with the adjacent samples.

The propused method was programed on MatLab R2007b and our test images (5.0 MPix each) were taken from test-olive-fields in Chile, performing very well with noisy pictures. About the computational complexity of the model, we run at an AMD Athlon(tm)X2 DualCore QL-66, 2.20GHz, 2,74 GB RAM that took just $83.3368 \mathrm{sec}$ each in a batch process. Moreover, dividing the model in three parts: segmentation, correlation and active contourns process they take $8.932 \%$, 3.088 and $87.980 \%$ of the average time respectivebly. 


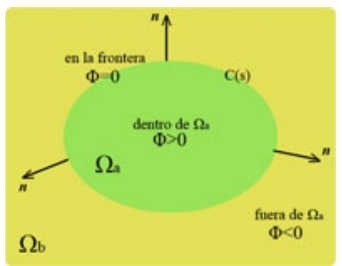

a)

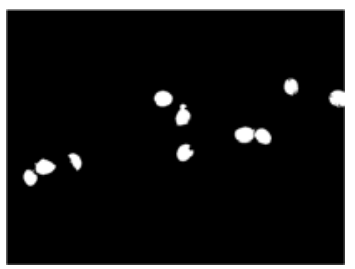

b)

Fig. 4. a) Chan-Vese Diagram; b) Result of active contours after 80 iterations

\section{Conclusions}

A model for the recognition of the diameter of olives has been presented. This allows estimating the best harvesting time for an olive tree. Recognition is performed by analyzing the RGB images obtained from olive tree pictures. By reviewing the related literature, all techniques used by many authors from around the world in the field were summarized. Review of past 30 years of publications show that there were no publications relating to the count of olives (olive fruits), only relating to the identification of olive trees (trees).

The proposed model allows not only to identify correctly the olives but also to measure millimeter caliber of each specimen. The idea of stratified data was used to investigate the possibility of utilizing stratification as the fundamental premise to identify that the CIELAB color space, specifically in the channel " $a$ ", could visually identify the possibility of a separation of layers and display a clear trend data normally.

Validation of data normality was performed by conducting a simple test of normality based on quadratic correlation between random variables and idealized normal density curves data, with excellent correlation of 0.9860 . Since the data is discrete and in set $\mathrm{Z}$, height of cut for the separation of strata is analyzed in terms of the distribution function, leaving the average height of cut 1.0441 standard deviations over the layer of olives. This theoretically ensures that the non-olive strata ought not to exceed $3 \%$ of incidence and an $85 \%$ for the layer of olives, leaving a random error of less than $3 \%$.

The success achieved in contour estimation was due largely to the proper choice of the number of iterations of the energy minimization algorithm. This selection was made empirically, concluding that for a great number of iterations the algorithm tends to re-assemble the contiguous contours, while for less iteration, the algorithm tends to underestimate the area of the olives.

\section{References}

1. Martinez-Uso, A., Pla, F., Garcia-Sevilla, P.: A Novel Energy Minimization Criterion for Color Image Segmentation. In: 17th Int. Conference on Pattern Recognition (ICPR 2004), vol. 3, pp. 206-209 (2004) 
2. Unay, D., Gosselin, B.: Artificial Neural Network-Based Segmentation And Apple Grading By Machine Vision. In: IEEE Int. Conference on Image Processing (ICIP 2005), vol. II-630-3 (2005)

3. Mohamad, F.S., Manaf, A.A., Chuprat, S.: Histogram Matching For Color Detection A Preliminary Study. In: Int. Symposium in Information Technology (ITSim), Kuala Lumpur, pp. 1679-1684 (2010)

4. Cornelius, H., Kragic, D., Eklundh, J.: Object and Pose Recognition Using Contour and Shape Information. In: Proc. 12th Int. Conference on Advanced Robotics (ICAR 2005), Seatle, WA, pp. 613-620 (2005)

5. Jin-jing, W., De-an, Z., Wei, J., Jun-jun, T., Ying, Z.: Application of Support Vector Machine to Apple Recognition using in Apple Harvesting Robot. In: IEEE Int. Conference on Information and Automation (ICIA 2009), Zuhuhai, Macau, pp. 1110-1115 (2009)

6. Zhao, J., Tow, J., Katupitiya, J.: On-tree Fruit Recognition Using Texture Properties and Color Data. In: IEEE/RSJ Int. Conference on Intelligent Robots and Systems (IROS 2005), pp. 263-268 (2005)

7. Jamil, N., Mohamed, A., Abdullah, S.: Automated Grading of Palm Oil Fresh Fruit Bunches (FFB) using Neuro-Fuzzy Technique. In: Int. Conference of Soft Computing and Pattern Recognition (SOCPAR 2009), Malaca, pp. 245-249 (2009)

8. Wijethunga, P., Samarasinghe, S., Kulasiri, D., Woodhead, I.: Digital Image Analysis Based Automated Kiwifruit Counting Technique. In: 23rd Int. Conference Image and Vision Computing New Zealand (IVCNZ 2008), Christchurch, pp. 1-6 (2008)

9. Song, W.-g., Guo, H.-x., Wang, Y.: A Method of Fruits Recognition Based on SIFT Characteristics Matching. In: Int. Conference on Artificial Intelligence and Computational Intelligence (AICI 2009), Shanghai, pp. 119-122 (2009)

10. Yinqing, L., Xiaodong, Z., Xiaojie, W.: Fruit Discrimination on Region Feature. In: Fifth International Conference on Fuzzy Systems and Knowledge Discovery (FSKD 2008), Shandong, pp. 590-594 (2008)

11. Saito, Y., Hatanaka, T., Uosaki, K., Shigeto, K.: Eggplant Classification Using Artificial Neural Network. In: Proc. of the International Joint Conference on Neural Networks, vol. 2, pp. 1013-1018 (2003)

12. Gatica, G.: A novel method for detection and counting of flower initiation in pear trees by hyperspectral and digital image analysis. Report at KU Leuven, Biosystems department, Belgium (2010)

13. Chan, T., Vese, L.: Active Contours Without Edges. IEEE Transactions on Image Processing 10(2) (2001)

14. Mumford, D., Shah, J.: Optimal approximations by piecewise smooth functions and associated variational problems. Comm. Pure Appl. Math. 42(5), 577-685 (1989)

15. Chan, T., Esedoglu, S.: A Multiscale Algorithm for Mumford-Shah Image Segmentation, Mathematics Department, UCLA (December 17, 2003)

16. Mery, M., Lefranc, G.: Computer Vision for Fruits Classification by Color and Size (in spanish). In: XVIII Congreso de la Asociación Chilena de Control Automático, Chile (December 2008)

17. Seng, W.C., Mirisaee, S.H.: A New Method for Fruits Recognition System. In: International Conference on Electrical Engineering and Informatics (ICEEI 2009), Selangor, vol. 1, pp. 130-134 (2009) 